\title{
Autophagy in Inflammatory Diseases
}

\author{
Alexander J. S. Choi ${ }^{1}$ and Stefan W. Ryter ${ }^{2,3}$ \\ ${ }^{1}$ College of Arts and Sciences, Boston College, 140 Commonwealth Avenue, Chestnut Hill, MA 02467, USA \\ ${ }^{2}$ Pulmonary and Critical Care Medicine, Brigham and Women's Hospital, Harvard Medical School, 75 Francis Street, \\ Boston, MA 02115, USA \\ ${ }^{3}$ Adjunct Scientist, Lovelace Respiratory Research Institute, Albuquerque, NM 87108, USA
}

Correspondence should be addressed to Stefan W. Ryter, sryter@partners.org

Received 9 August 2011; Accepted 14 September 2011

Academic Editor: J. Chloe Bulinski

Copyright (C) 2011 A. J. S. Choi and S. W. Ryter. This is an open access article distributed under the Creative Commons Attribution License, which permits unrestricted use, distribution, and reproduction in any medium, provided the original work is properly cited.

\begin{abstract}
Autophagy provides a mechanism for the turnover of cellular organelles and proteins through a lysosome-dependent degradation pathway. During starvation, autophagy exerts a homeostatic function that promotes cell survival by recycling metabolic precursors. Additionally, autophagy can interact with other vital processes such as programmed cell death, inflammation, and adaptive immune mechanisms, and thereby potentially influence disease pathogenesis. Macrophages deficient in autophagic proteins display enhanced caspase-1-dependent proinflammatory cytokine production and the activation of the inflammasome. Autophagy provides a functional role in infectious diseases and sepsis by promoting intracellular bacterial clearance. Mutations in autophagy-related genes, leading to loss of autophagic function, have been implicated in the pathogenesis of Crohn's disease. Furthermore, autophagy-dependent mechanisms have been proposed in the pathogenesis of several pulmonary diseases that involve inflammation, including cystic fibrosis and pulmonary hypertension. Strategies aimed at modulating autophagy may lead to therapeutic interventions for diseases associated with inflammation.
\end{abstract}

\section{Introduction}

1.1. Inflammation. Acute inflammation acts as part of the host's innate protective response to infection or tissue injury. Endothelial cell injury or microbial infection causes changes in vascular permeability, local edema, and in the distribution of chemoattractants $[1,2]$. The activation of endothelial cells allows the transmigration of leukocytes, initially primarily neutrophils (polymorphonuclear (PMN) cells), to the site of tissue injury [3]. Finally, macrophages uptake apoptotic PMN cells, cellular debris, and invasive pathogens via phagocytosis during the resolution of acute inflammation, which leads to neutrophil clearance and the release of anti-inflammatory cytokines such as transforming growth factor- $\beta 1$. The resolution program ends with the efflux of macrophages from the site of inflammation through lymphatics [4]. However, aberrant inflammatory responses can be associated with a wide range of acute, chronic, and systemic inflammatory disorders, such as cardiovascular disease, asthma, inflammatory bowel disease, rheumatoid arthritis [1], and cystic fibrosis [5].

In recent years, emerging evidence has indicated that the process of macroautophagy may play an essential role for the host during bacterial clearance [6] as well as interact with inflammatory processes, and thereby potentially impact the outcome of disease progression.

1.2. Autophagy. Macroautophagy (autophagy) refers to the cellular pathway for the degradation or disposal of organelles and proteins via lysosomal processes. The autophagy mechanism involves double-membrane vesicles, called autophagosomes or autophagic vacuoles (AVs) that target and engulf cytosolic material, which may include damaged organelles or denatured proteins. The autophagosomes fuse with lysosomes to form single-membrane autolysosomes. Lysosomal enzymes facilitate this degradation process to regenerate metabolic precursor molecules (i.e., amino acids and fatty 
acids), which can be used for anabolic pathways and energy production [7-12]. This process may thereby prolong cellular survival during starvation. During infection, autophagy assists in the immune response by providing a mechanism for the intracellular degradation of invading pathogens such as bacteria [5]. Furthermore, autophagy influences the immune system during pathogen clearance by regulating antigen presentation, lymphocyte development, and proinflammatory cytokine production [13]. However, the mechanism for the involvement of autophagy in cytokine secretion remains poorly understood. In addition to macroautophagy, several other subtypes of autophagy exist, including microautophagy and chaperone-mediated autophagy [14].

At least 30 autophagy-related (Atg) genes have been determined, primarily in yeast. The homologues of many of these Atg genes have been shown to participate in the regulation of autophagy [14-16]. Among these, Beclin 1 (the mammalian homolog of yeast Atg6) represents a major autophagic regulator and tumor suppressor protein [17].

Recent studies suggest that autophagy evolved as a homeostatic response for unicellular eukaryotic organisms. Moreover, the same autophagy process could be used for diverse functions in more complex multicellular organisms in response to various stressful stimuli [5]. Therefore, the evolving understanding of autophagy and its interaction with other intracellular processes may reshape our knowledge and lead to the development of therapies for inflammatory disorders.

Recent studies suggest that the process of autophagy may be more selective than originally described such that there exist specific molecular mechanisms that regulate the autophagy-dependent intracellular degradation of bacteria, denatured protein aggregates, mitochondria, and other subcellular substrates [18]. Autophagy plays an important role in the maintanance of healthy organelle populations by eliminating damaged specimens (e.g., mitochondria and endoplasmic reticulum (ER)).

In addition to providing basic homeostatic functions, autophagy can potentially impact other vital cellular processes, including programmed cell death (i.e., apoptosis). The complex relationship between autophagy and cell death pathways has been reviewed elsewhere [19-21]. It is now well recognized that autophagy can exert a critical influence on systemic immune and inflammatory responses and on the specific cell types that mediate these responses. This paper will summarize how these dynamic relationships influence the pathogenesis of several diseases, including pulmonary and systemic diseases, where inflammatory processes have been implicated.

1.3. Autophagic Machinery. The activation of the autophagic pathway involves the assembly of several macromolecular signaling complexes $[14,16]$. These include the mammalian target of rapamycin (mTOR) complex-1 (mTORC1) which consists of mTOR and several accessory proteins. The mTORC1 regulates a macromolecular substrate complex (mTOR substrate complex) containing the mammalian uncoordinated-51-like protein kinase ULK1 (the mammalian homolog of yeast Atg1) and other factors (i.e., Atg13, FIP200, and Atg101) [22-25]. The mTOR pathway negatively regulates autophagy during nutrient-rich conditions. Through this pathway, starvation and stimulation with the immunosuppressive drug rapamycin potently induce autophagy, by inhibiting mTOR kinase activity, and thereby permitting the activation of ULK1 kinase, a major initiator of autophagy $[26,27]$.

The de novo formation of the autophagosome membrane, presumably originating from the ER, requires a major macromolecular regulatory complex that includes Beclin 1 and Vps34, a Class III phosphatidylinositol-3-kinase (PIK3C3) [28]. Several additional proteins can interact with and influence the activity of this complex (e.g., Rubicon, UVRAG, and Bcl-2 family proteins, etc.). Activation of PIK3C3 activity leads to the increased production of phosphatidylinositol-3-phosphate (PI-3P) which regulates the initial steps in autophagosome formation [28].

The subsequent elongation of autophagosomes requires the activation of two ubiquitin-like conjugation systems [14, 16, 30]. First, the ubiquitin-like protein Atg12 is conjugated to Atg5 by Atg7 (E1-like) and Atg10 (E2-like) enzymes. The Atg5-Atg12 complex in turn associates with Atg16L (the mammalian homolog of yeast Atg16). The resulting multimeric complex assists in the elongation of the autophagic membrane [30].

A second conjugation system requires the ubiquitinlike protein, microtubule-associated protein-1 light chain 3 (LC3), and the mammalian homologue of yeast Atg8 [31, 32]. Several homologues of LC3 (i.e., LC3B) and related cellular cognate proteins (i.e., GABARAP) are conjugated with the phospholipid phosphatidylethanolamine (PE) [32, 33]. Atg4 B catalyzes the proteolytic processing the LC3 proform to generate the cleaved form LC3-1. Conjugation of LC3-I with PE is subsequently catalyzed by Atg7 (E1-like) and Atg3 (E2-like) activities [30]. In mammals, the conversion of LC3I (unconjugated form) to LC3-II (lipidated form) is generally regarded as a key regulatory step and indicator of autophagosome formation [32]. In the final stages of autophagy, the autophagosome matures and fuses with the lysosome, where encapsulated cargoes are digested by resident hydrolase activities [9]. Autophagosome maturation and fusion are assisted by several additional regulatory proteins, including small GTPases and lysosome-associated membrane proteins (i.e., LAMP2) $[14,34,35]$.

Recently, additional proteins (i.e., p62 ${ }^{\text {SQSTM1}}$, NBR1, NDP52, Nix, and others) have been identified in the selection of autophagic cargo. Collectively, these proteins, which are selectively degraded by autophagosomes, act as autophagic adaptors, or cargo receptors for prospective cargos including ubiquitinated proteins, bacteria, or mitochondria [18]. To be considered as an active process, autophagy requires the completion of all steps of the autophagic pathway including substrate turnover, which are collectively referred to as autophagic flux [35]. Changes in LC3B expression and/or conversion, or accumulations of autophagosome numbers, do not necessarily represent active autophagy, as these conditions can arise if the autophagosome-lysosome fusion event and/ or subsequent lysosomal processing steps are blocked or impaired [36, 37] (Figure 1). 


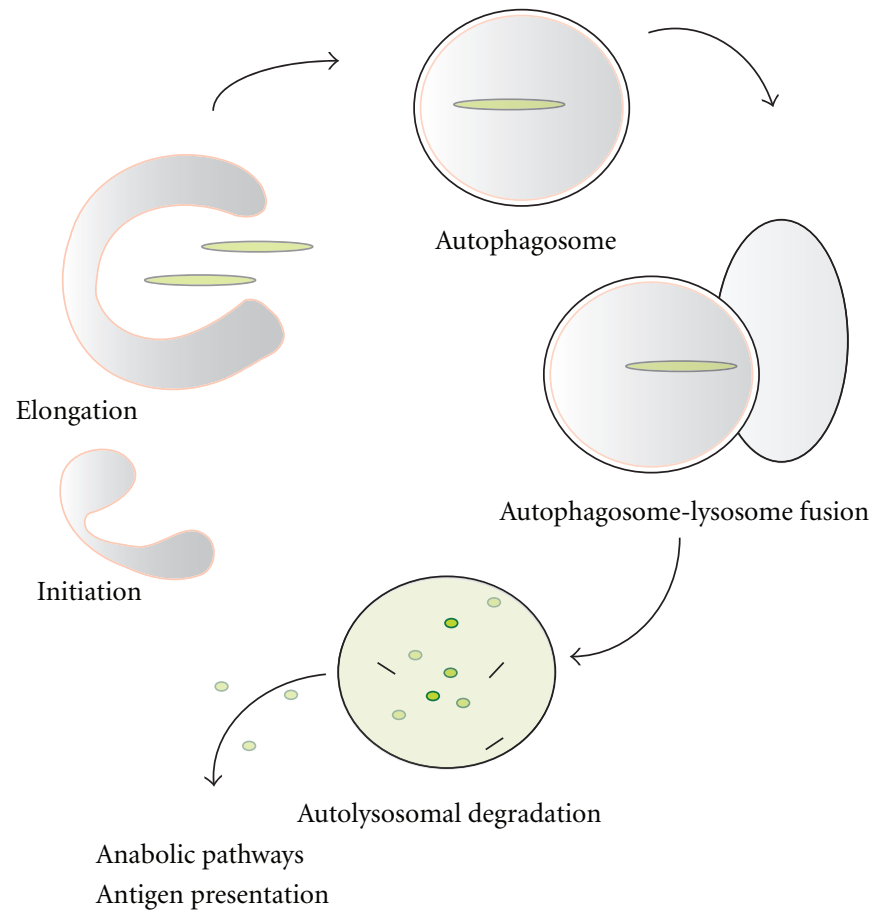

FIGURE 1: The "macroautophagic" pathway responds to stimulation by various environmental cues including nutrient availability or noxious agents, which result in the accumulation of damaged proteins and/or organelles as well as pathogenic bacteria or viral infection. In the nucleation phase, a preautophagosomal structure develops from subcellular membranes and subsequently evolves into the phagophore or isolation membrane. The isolation membrane then expands to surround and engulf a cytoplasmic "cargo" of material targeted for degradation, culminating in double-membraned autophagosomes. Finally, the fusion of autophagosomes with lysosomes results in the formation of the autolysosome. During the degradative phase of autophagy, the encapsulated contents of autolysosomes are digested by lysosomal degradative enzymes (e.g., cathepsins and other acid hydrolases). The digested contents are then released to the cytosol for reutilization in anabolic pathways.

\section{Interaction of Autophagy with Inflammation and Immune Responses}

2.1. Autophagy and Inflammatory Signaling. The signaling pathways that regulate inflammatory processes now apparently have a role in the regulation of autophagy and viceversa. In addition to classical signals such as starvation and energy exhaustion, several pathogen-associated molecular patterns (PAMPs) have been shown to promote autophagic activation [6]. Recent studies suggest that Toll-like receptors (TLR), the primary cellular sensors for PAMPs, can regulate autophagy through the activation of downstream signaling processes in macrophages and other cells types (reviewed in reference [6]). For example, the TLR9 ligand, bacterial CpG motifs, can induce autophagy in rodent and human tumor cell lines [38]. The screening of TLR ligands for their capacity to induce LC3 puncta (in green fluorescent proteinLC3 assays) revealed that single-stranded RNA (ssRNA) and imiquimod, two model TLR7 ligands, were relatively potent inducers of autophagy [39]. Bacterial LPS, a TLR4 ligand, has been implicated in several studies as a stimulator of autophagic signaling in cultured macrophage cell lines [39, 40]. The ability of LPS to induce autophagy in primary macrophages, however, has been disputed [41].
Additional studies suggest that autophagic proteins can modulate responses to viral infections. For example, the IFN- $\beta$ response to double-stranded DNA (dsDNA) is enhanced in mouse embryonic fibroblasts by genetic deletion of Atg9a [42]. For example, the deletion of Atg5 in macrophages and murine embryo fibroblasts was reported to enhance type 1 interferon production in response to ssRNA virus [43]. The amplification of IFN responses to infection in these cell types was attributed to loss of mitochondrial quality control and enhanced mitochondrial ROS production in response to impaired autophagic processing [43]. In contrast, independent studies reported that the IFN- $\alpha$ response to infection with ssRNA virus (i.e., vesicular stomatitis virus) was compromised in Atg5 $5^{-/-}$chimeric mice and in $A \operatorname{tg} 5^{-/-}$ dendritic cells [44]. Independently of autophagy, Atg5 was also shown to play a role in resistance to the intracellular pathogen Toxoplasma gondi by facilitating the recruitment of a p47 GTPase to the bacteria containing vacuole [45].

Recent observations have revealed a relationship between autophagic proteins and inflammasome-associated proinflammatory cytokine maturation in macrophages [41, 46, 47]. Inflammasomes are cytosolic multiprotein complexes that constitute a novel inflammatory signaling mechanism and which govern the maturation and secretion of select 
proinflammatory cytokines, such as IL-1 $\beta$, IL-18, and IL-33 [48]. Cytosolic receptors of the NOD-like receptor (NLR) family (i.e., NLRP3 and NLRP1) interact with binding partners to form inflammasome complexes. NLRP3 interacts with an adaptor protein (apoptosis-associated speck like protein containing CARD (ASC)), which recruits and activates the procaspase- 1 by proteolytic cleavage [48].

Proinflammatory cytokine secretion (IL-1 $\beta$ and IL-18) was enhanced in atg16l1 or atg7 deleted macrophages in response to LPS [41]. In contrast, atg16l1 or atg7 deficiency did not affect TNF and IFN- $\beta$ production or NF- $\kappa$ B pathway activation in macrophages stimulated with LPS [41]. Furthermore, atg16l1-deleted mice displayed increased susceptibility to a murine model of colitis, which could be ameliorated by anti-IL-18 therapy [41]. In recent studies, increased activation of IL- $1 \beta$ and IL-18 has also been observed in macrophages and monocytes isolated from mice genetically deficient in Beclin 1 and LC3B [46].

Cytokine activation in response to LPS and ATP in wildtype macrophages, as well as the amplification observed in LC3B or Beclin 1-deficient macrophages, required the NLRP3 inflammasome pathway $[46,47]$. The mechanism by which autophagy deficiency enhanced NLRP3 inflammasome pathway activation was mediated by deregulation of mitochondrial homeostasis, including the enhanced production of mitochondrial ROS and increased mitochondrial membrane permeability transition $[46,47]$. The pathway to caspase-1-dependent IL-18 secretion in macrophages was further shown to be blocked by mitochondrial targeting antioxidants [46]. These experiments, taken together, suggest that autophagic proteins dampen inflammasome pathway activation by stabilizing mitochondria and/or maintaining mitochondrial quality control through autophagy. Further research in this area may uncover additional mechanisms. Taken together these studies suggest an important role for autophagic proteins in the dampening of proinflammatory responses, and that warrants further investigation in models of inflammatory disease.

2.2. Autophagy and Adaptive Immunity. Autophagy plays critical role in bacterial clearance mediated by autophagosomal sequestration and subsequent autolysosome-dependent degradation and in the regulation of the cytokine response [5] (Figure 2). In addition to these roles, recent studies also indicate that autophagy can participate in adaptive immune responses, including antigen presentation, and in the maintenance of lymphocyte function [5, 49]. The discovery that autophagosomes can fuse with and transfer content to major histocompatibility complex (MHC) Class II loading compartments illustrates the importance of this relationship [50]. The immune system detects pathogen-derived antigens (i.e., peptides) and initiates a response through MHC Class I and II loading compartments. The peptide fragments generated by intracellular degradation of bacteria, including autolysosomal degradation, are displayed on MHC Class I and II molecules. Class I MHC molecules are generally present in most cell types and assist in antigen presentation to $\mathrm{CD}^{+} \mathrm{T}$ cells. Inhibition of autophagy by chemical inhibitors or genetic knockdown of select autophagic proteins (i.e., Atg5) typically does not affect MHC Class I antigen presentation [49]. However, autophagy induction in target cells was shown to increase their ability to act as immunogens for dendritic cell cross-presentation to $\mathrm{CD}^{+} \mathrm{T}$ cells [51]. Furthermore, autophagy induced during HSV-1 infection enhances the presentation of viral-derived antigen on $\mathrm{MHC}$ Class I molecules [52]. Class II MHC molecules (which are found specifically in antigen-presenting cells such as macrophages, B cells, and dendritic cells) present bacterial fragments to $\mathrm{CD} 4{ }^{+} \mathrm{T}$ cells, which mediate immune responses from other cell types. Genetic interference of Atg12 was shown to inhibit MHC Class II antigen presentation to CD $4^{+}$ $\mathrm{T}$ cells during Epstein-Barr virus infection [53]. Genetic deletion of Atg5 also suppressed the processing and presentation of herpes simplex virus-2- (HSV-2-) derived antigen on MHC Class II molecules and enhanced vulnerability of mice to HSV-2 infection [54]. Recent studies have identified a novel role for autophagy in the generation of a self-tolerant $\mathrm{T}$ cell repertoire. Constitutively, highly expressed autophagy in thymic epithelial cells delivers endogenous proteins to MHC Class II molecules and contributes to $\mathrm{CD}^{+} \mathrm{T}$ cell selection. The grafting of embryonic thymi from $A \operatorname{tg} 5^{-/-}$ mice into athymic nude mice was shown to promote systemic lymphoid infiltration [55]. Taken together, these examples suggest that autophagy and/or autophagic proteins play multivariate roles in immune system function.

\section{Autophagy in Inflammatory Diseases}

3.1. Autophagy in Crohn's Disease. Crohn's disease is a chronic inflammatory bowel disease characterized by inflammation, ulceration, and neutrophil influx in the intestinal epithelia. The pathophysiological mechanisms of Crohn's disease remain unclear but may involve excess inflammatory responses, abnormal Paneth cell granule secretion, and impaired intracellular bacterial clearance [56]. Recent human studies have suggested links between autophagy and Crohn's diseases. Genome-wide association studies (GWAS) have revealed small nucleotide polymorphisms (SNPs) in autophagy genes such as ATG16lL and in additional genes now known to influence autophagic processing (i.e., NOD2 and IRGM) associated with susceptibility to Crohn's disease [57-61].

The first of these to be described, a T300A variant in the ATG16lL gene, has been identified as an associated risk factor for Crohn's disease. ATG16lL plays a key role in autophagosome formation [57, 58]. Genetic deletion of ATG16lL impairs autophagosome formation and autophagic processing of protein and, furthermore, promotes IL- $1 \beta$ production in macrophages in response to LPS stimulation [41]. The mechanism by which the T300A mutation in ATG16lL compromises autophagic function remains unclear [6].

Variants in the gene encoding immunity-related p47 guanosine triphosphatase (IRGM) were associated with Crohn's disease in a recent GWAS [62]. Its murine homologue Irgm1 can regulate intracellular autophagy in response to IFN- $\gamma$ stimulation and starvation. Mice rendered deficient in $\operatorname{Irgm} 1$ 


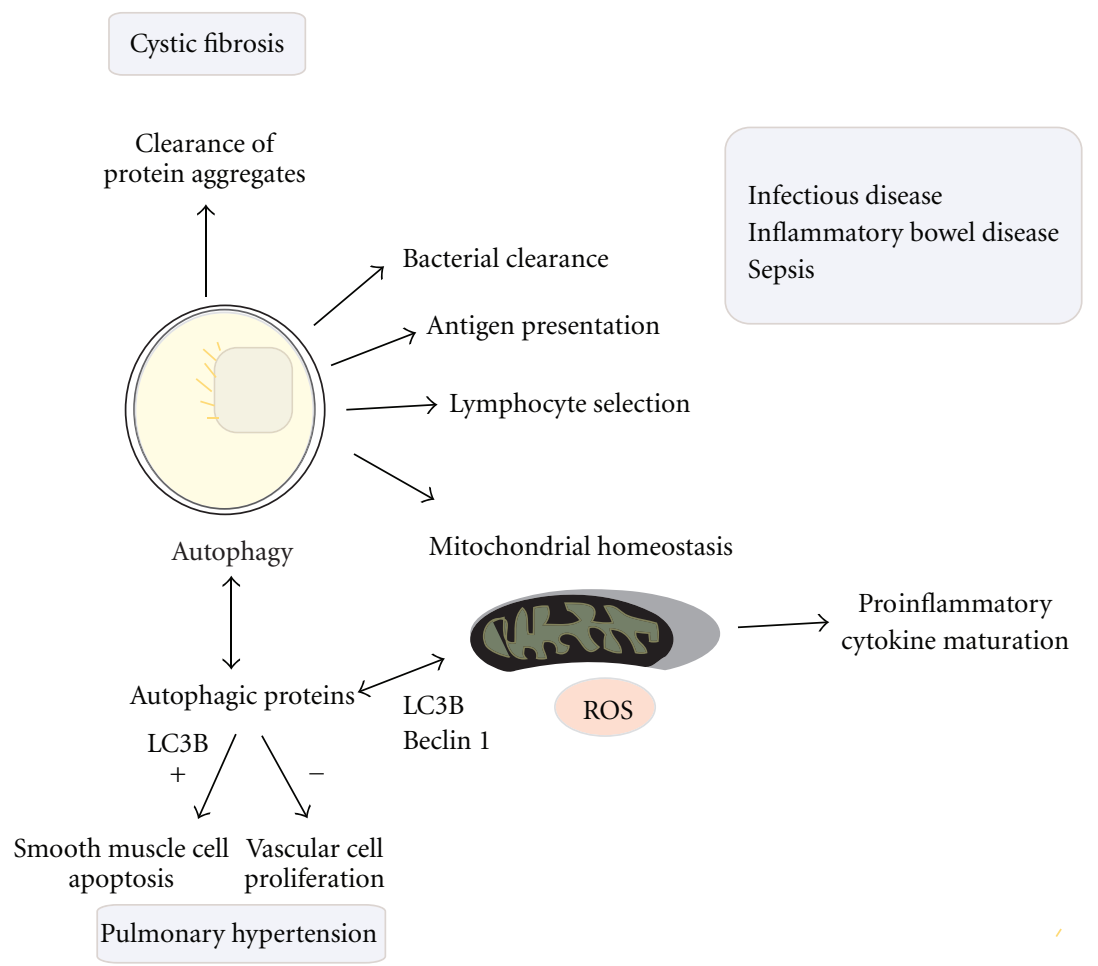

FIGURE 2: Autophagy as an adaptive cellular process potentially impacts the progression of inflammatory diseases by several possible mechanisms. (i) Autophagy, by acting as a "xenophagic" response, directly participates in bacterial clearance, through the encapsulation and lysosomal delivery of invading bacteria for degradation. (ii) Autophagic processes can assist in antigen presentation through the digestion of invading pathogens. (iii) Autophagic proteins play a role in the dampening proinflammatory responses, including proinflammatory cytokine secretion, through the maintenance of mitochondrial quality. (iv) Autophagic degradation of denatured protein aggregates may play a protective role in tissues such that impaired function has been associated with diseases such as cystic fibrosis [5]. (v) Autophagic protein LC3B potentially regulates other cellular processes as recently described in a model of hypoxia-induced pulmonary hypertension. In these studies, LC3B was found to inhibit vascular cell proliferation and promote smooth muscle cell apoptosis, collectively associated with protection in this model [29].

displayed an impaired ability to clear intracellular bacteria [63]. Recent studies suggest that human IRGM regulates autophagy through dynamic interactions with mitochondria $[64,65]$. IRGM associates with mitochondria by binding to the phospholipid cardiolipin, a constituent of the mitochondrial inner membrane, and consequently promotes mitochondrial membrane depolarization and mitochondrial fission [65].

At least three mutations in the gene encoding nucleotide-binding oligomerization domain 2 (NOD2), also known as CARD15, including small nucleotide polymorphisms (R702W and G908R) and a frameshift mutation (L1007fsinsC) have been found in association with Crohn's disease [66-73]. NOD2, a protein of the NLR family, functions as an intracellular bacteria sensor. NOD2 activates signaling pathways in response to stimulation with bacteriaderived peptides [74]. Muramyl dipeptide (MDP), a component of the bacterial peptidoglycan cell wall, induces autophagy in intestinal epithelial cells, and thereby also promotes autophagy-dependent bacterial clearance [75]. The induction of autophagy by MDP requires NOD2 and ATG16L and involves physical interaction of NOD2 with ATG16L [76].
NOD2 recruits ATG16L to the membrane site of bacterial entry and facilitates association of LC3B with bacteria [76]. The expression of NOD2 genetic variants associated with Crohn's disease results in impaired autophagic processing of pathogens (i.e., Salmonella typhimurium) by epithelial cells in response to treatment with NOD2 ligand [75]. In addition to stimulation of autophagy-dependent bacterial clearance, NOD2 was recently shown to also regulate dendritic cell MHC Class 2 dependent antigen presentation to $\mathrm{CD}^{+}{ }^{+} \mathrm{T}-$ cells [77]. Recent studies also show that inhibition of the autophagy process or expression of the ATG16IL T300A variant leads to increased proinflammatory cytokine responses (i.e., IL-1 $\beta$ and IL-6) in human primary immune cells in response to stimulation with NOD2 ligands [78].

Taken together, defects of autophagic activity as the result of mutations in autophagy-associated genes (i.e., ATG16L1 and IRGM) and bacterial sensors (NOD2) have been associated with the impaired clearance of harmful bacterial species associated with Crohn's disease, impaired antigen presentation, and also with the higher production of proinflammatory cytokines implicated in the pathogenesis of Crohn's disease. 
3.2. Autophagy in Respiratory Infections. Autophagy can exert antibacterial and antipathogen functions, which were originally demonstrated in several infectious disease models using live bacteria $[79,80]$. The general role of autophagy in host defense against various microbes including bacteria, viruses, and parasites has now been widely recognized [6].

Phagocytosis of nonpathogenic mycobacteria by macrophages leads to autophagy and apoptosis, which results in the termination of the microbe. However, phagocytosis of pathogenic mycobacteria inhibits the autophagy pathway with the acidification of phagosomes and lysosomal fusion [81]. Mutations in NOD2, a pathogen-recognition receptor critical for bacterial autophagy $[76,77]$, are associated with vulnerability to microbial infection with the etiologic agent of leprosy, Mycobacterium leprae [82].

In the case of $M$. tuberculosis, the mycobacteria remains and replicates in immature phagosomes. In addition, instead of stimulating macrophage apoptosis, phagocytosis of $M$. tuberculosis promotes necrotic cell death, which promotes bacteria dispersal to uninfected cells. As a result, reduced mycobacterial antigen presentation and chronic M. tuberculosis infection occur [83]. However, experimental stimulation of autophagy can reduce intracellular replication and survival of $M$. tuberculosis [79, 83-85]. Conversely, chemical inhibitors of autophagy promote infection $[80,86]$.

IFN- $\gamma$ production makes an important contribution to host defense against $M$. tuberculosis. Macrophages stimulated with IFN- $\gamma$ induce autophagy, and this response facilitates the resolution of infection [80, 87]. IFN- $\gamma$-stimulation can thereby bypass the inhibition of lysosomal fusion of virus containing phagosomes, leading to the destruction of the organism by $162^{\text {SQSTM1 }}$-dependent selective autophagy [87], and resolution of infection. IFN- $\gamma$ induced autophagy involves the p47 guanosine triphosphatase IRGM-1 [64, $65,80,88]$. Interestingly, small nucleotide polymorphisms occurring in the IRGM-1 gene, as implicated in Crohn's disease, were recently also linked to increased susceptibility to M. tuberculosis infection $[89,90]$. Finally, autophagic process may assist in the generation of antivirulence factors against this organism through degradation of substrate proteins $[87,91]$.

Given that M. tuberculosis is the pathogenic agent causing tuberculosis, this bacterium is a major contributor to global disease burden [92]. Therefore, therapeutic strategies involving autophagy pathway manipulation to reduce infection and promote adaptive immunity to this organism, and to other related pathogens, may be of considerable interest. Additional studies implicate autophagy in defense against other respiratory pathogens, such as Legionella pneumophila, the causative agent in Legionnaire's disease. For example, genetic deficiency of atg 9 was shown to promote the growth of Legionella pneumophila, which suggests a role of autophagy in defense against this organism $[93,94]$.

Recent studies have shown that the fundamental mechanisms of host pathogenic response are conserved in lower eukaryotes [95-97]. Also, autophagy remains critical for normal cellular development of the social amoeba in Dictyostelium discoideum [98]. Therefore, D. discoideum has become a widely used model system for the study of bacterial infection and autophagy [99-101]. Infection of D. discoideum with Legionella pneumophila causes a large increase in differentially regulated autophagy-related genes, including ATG8, ATG9, and ATG16 [102]. However, recent studies have shown that L. pneumophila can undergo replication in autophagy mutants of $D$. discoideum [103].

3.3. Autophagy in Sepsis. Sepsis remains a leading cause of mortality in intensive care units. This condition arises as a consequence of systemic responses to inflammation caused by acquired bacterial, fungal, parasitic, or viral infections and may lead to multiple organ failure [104].

To date, little is known of the role of autophagy in sepsis. Marked autophagosome accumulation has been observed in the livers of patients who die from sepsis [105]. However, it currently remains unclear whether this observation represents increased autophagic activity (flux) in sepsis patients or inhibition of autophagic processing which leads to the inappropriate accumulation of autophagosomes. Genetic deletion of critical autophagic proteins has recently been shown to increase sepsis-induced inflammatory responses in mice subjected to the cecal-ligation and puncture (CLP) model of polymicrobial sepsis [46]. Similar results were observed in mice challenged with LPS injection [46]. Furthermore, $B E C N 1^{+/-}$mice and $L C 3 B^{-/-}$mice were found to be susceptible to the lethal effects septic shock in mice, and to express higher levels of IL-18, one of the inflammasome-associated cytokines in the plasma [46]. Collectively, these studies suggest a potential link between autophagy and inflammatory responses during the pathogenesis of sepsis.

\section{Autophagy in Pulmonary Disease}

Recent evidence from this laboratory and other, suggests that autophagy may be critically involved in other noninfectious pulmonary diseases, where inflammation has been implicated. In these cases, additional functional aspects of autophagy may indirectly affect inflammation by limiting tissue injury. In two illustrative examples, we describe recent work illustrating how autophagy may impact the pathogenesis of pulmonary hypertension and cystic fibrosis.

\subsection{Role of Autophagic Protein LC3B in Pulmonary Hyper-} tension. Recent studies from this laboratory have sought to determine the involvement of autophagic proteins in pulmonary arterial hypertension (PAH). PAH is a complex disease of varying etiologies which include idiopathic and forms as well as other subtypes (i.e., associated with left heart disease, HIV infection, etc.). PAH is characterized mainly by vasoconstriction, increased pulmonary artery pressure, thickening and fibrosis of the artery, which may lead to cardiac dysfunction, and right ventricular hypertrophy [106, 107]. We examined the prospective role of autophagic proteins in an experimental mouse model of chronic hypoxia-induced pulmonary hypertension. Exposure to chronic hypoxia in mice resulted in the increased expression of LC3B and its conversion of LC3B-II in the lung. Increased LC3B staining was also observed in 
small pulmonary vessels of animals subjected to hypoxia. Moreover, hypoxic lungs contained elevated numbers of autophagosomes, as detected by electron microscopy. Importantly, mice genetically deleted for $\operatorname{LC} 3 \mathrm{~B}\left(\mathrm{LC}_{\mathrm{B}} \mathrm{B}^{-/-}\right)$displayed increased indices of pulmonary hypertension, including increased right ventricular systolic pressure, and Fulton's Index relative to wild-type mice, after chronic hypoxia [29]. These results identified an endogenous role for autophagic protein $\mathrm{LC} 3 \mathrm{~B}$ in the regulation of protective processes during the development of pulmonary hypertension. Genetic deletion of LC3B aggravated the hypertensive phenotype as the result of hypoxia exposure. These observations were corroborated with observations in vitro of increased vascular cell (i.e., endothelial and smooth muscle) proliferation and impaired smooth muscle cell apoptosis in after LC3B-specific genetic knockdown. These experiments, which have used LC3B knockout or knockdown strategies, suggest a specific role for the autophagic protein LC3B, in vascular responses to hypoxia, and associated pathogenic processes implicated in the development of pulmonary hypertension. However, these experiments did not unequivocally establish a specific role for autophagic activity in these phenotypes. The authors could not exclude that LC3B exerts effects on signaling processes independently of the process of autophagy. In contrast, the vascular changes recorded in Beclin $1^{+/-}$mice were not statistically significant. Experiments using additional autophagy protein knockout mice (i.e., Atg5) may be warranted.

The relevance of these findings to clinical disease, was supported by similar observations in human tissues from patients with pulmonary hypertension. Human lung tissue isolated from patients with pulmonary hypertension $(\mathrm{PH})$ of various etiologies, including $\mathrm{PAH}$, displayed increases in the total expression of LC3B, and in the levels of its activated (PEconjugated) form LC3B-II, when compared to lung tissue from patients free of pulmonary vascular disease. The expression of LC3B was markedly increased in the endothelial cell layer, as well as in the adventitial and medial regions of large and small pulmonary resistance vessels from $\mathrm{PH}$ lung, relative to normal vascular tissue. These results, taken together, suggest that autophagic proteins may potentially be exploited for the prevention and/or treatment of vascular disease in humans [29].

4.2. Autophagy in Cystic Fibrosis: A Role for "Aggrephagy". In addition to effects on bacterial clearance and the resolution of inflammation, autophagy may exert additional functions that could ameliorate inflammatory diseases. One of these functions is the selective clearance of aggregated and denatured protein, a process termed "aggrephagy". This function is exemplified by recent studies on the role of autophagy in cystic fibrosis (CF), a debilitating autosomal recessive disorder. CF patients are marked by the collection of misfolded proteins in the airway epithelia due to mutations ( $\Delta$ F508 and others) in the gene encoding the cystic fibrosis transmembrane conductance regulator (CFTR) [1].

The pathological features of CF include aberrant accumulation of hyperviscous mucous in the airways, impaired mucociliary clearance, and increased inflammation. Lung injury may also arise from secondary infections (i.e., Staphylococcus aureus, Pseudomonas aeruginosa, etc.) [108].

Recent studies demonstrate that human airway epithelial cells from CF patients, which bear the mutation in the CFTR gene, have an impaired autophagic response. In response to starvation, a classical inducer of autophagy, these cells exhibited reduced autophagosome formation and accumulation of $\mathrm{p} 62^{\text {SQSTM1 }}$. The human epithelial cells with CFTR mutation also displayed an abnormal accumulation of polyubiquitinated protein aggregates indicative of impaired aggresome clearance. In normal epithelial cells, mutation and/or loss of function of CFTR was associated with elevated reactive oxygen species (ROS) production and increased tissue transglutaminase 2 (TG2) levels, an important factor of inflammatory response in CF. Activation of these pathways caused a loss of function in Beclin 1 and the Beclin 1/PIK3C3 complex and, as a result, loss of autophagic function. Beclin 1 overexpression, or the application of cystamine or other antioxidants restored Beclin 1 function and autophagy and reverted the CF airway phenotype in human CF nasal biopsies, in vivo in Scnn1b-transgenic mice (a model of $\mathrm{CF}$ ), and CftrF508del homozygous mice as well as in cells expressing mutant CFTR $(\Delta \mathrm{F} 508)$ in vitro. Reconstitution of Beclin 1 levels also restored the membrane trafficking of mutant CFTR and reduced its accumulation in aggresomes. Furthermore, recent studies have shown that the accumulation of p62 $2^{\text {SQSTM1 }}$, an LC3-binding and ubiquitin-binding protein, in the context of impaired autophagy, promotes the aberrant accumulation of intracellular protein aggregates in human CF airway epithelial IB3-1 cells. Thus, defective CFTR causes impaired autophagy processing, which favors the accumulation of aggresomes, and lung inflammation $[5,109]$. In conclusion, selective targeting the autophagic pathway may be included in the design of therapeutics for the treatment of CF.

\section{Final Remarks}

Current studies indicate that autophagic processes can exert a significant impact on the regulation of inflammation, on the resolution of infection, and on immune responses to invading pathogens. These observations collectively implicate autophagy as an important modulator of disease pathogenesis. The bacterial clearing function of autophagy may contribute to host defenses in diseases involving bacteria, such as sepsis, inflammatory diseases of the bowel, and respiratory infections. Furthermore, autophagy may serve a function in downregulating proinflammatory cytokine production implicated in tissue injury, which may also exert a protective role in inflammatory diseases not necessarily involving bacterial infection. The ability of autophagy to clear aggregated protein (i.e., aggrephagy) as well as to maintain mitochondrial homeostasis (i.e., mitophagy) may also play supporting roles in protection against diseases associated with inflammation. Finally, the possibility remains that autophagic proteins may regulate cellular processes independently of their role in regulating autophagic activation. Much progress has accumulated in understanding these 
relationships in select infectious and inflammatory diseases. Further research will determine whether the autophagic pathway can be manipulated for therapeutic gain in the treatment of inflammatory diseases and/or other diseases of the lung and cardiovascular system $[110,111]$.

\section{Abbreviations}

$\begin{array}{ll}\text { ATG: } & \text { Autophagy-related gene } \\ \text { CF: } & \text { Cystic fibrosis } \\ \text { CFTR: } & \begin{array}{l}\text { Cystic fibrosis transmembrane conductance } \\ \text { regulator }\end{array} \\ \text { CLP: } & \text { Cecal ligation and puncture } \\ \text { GWAS: } & \text { Genome-wide association study } \\ \text { IFN: } & \text { Interferon } \\ \text { IRGM: } & \text { Immunity-related p47 guanosine } \\ & \text { triphosphatase } \\ \text { LC3B: } & \text { Microtubule-associated protein-1 light chain } \\ & \text { 3 } \\ \text { LPS: } & \text { Lipopolysaccharide } \\ \text { mTOR: } & \text { Mammalian target of rapamycin } \\ \text { mTORC1: } & \text { mTOR complex 1 } \\ \text { MHC: } & \text { Major histocompatibility complex } \\ \text { NLR: } & \text { NOD-like receptor } \\ \text { NLRP3: } & \text { NOD-like receptor protein-3 } \\ \text { NOD2: } & \text { Nucleotide-binding oligomerization domain } 2 \\ \text { p62SQSTM1 } & \text { 62-kDa protein, selective autophagy substrate } \\ \text { PAH: } & \text { Pulmonary arterial hypertension } \\ \text { PH: } & \text { Pulmonary hypertension } \\ \text { PIK3C3: } & \text { Phosphatydylinositol-3-kinase (Class 3) } \\ \text { PMN: } & \text { Polymorphonuclear cells } \\ \text { ROS: } & \text { Reactive oxygen species } \\ \text { SNP: } & \text { Small nucleotide polymorphism } \\ \text { ssRNA: } & \text { Single stranded RNA } \\ \text { TLR: } & \text { Toll-like receptor } \\ \text { ULK-1: } & \text { Uncoordinated-51-like protein kinase. } \\ & \\ & \end{array}$

\section{Acknowledgments}

This work was performed as part of a joint educational program between Boston College and Brigham and Women's Hospital (BWH), Harvard Medical School. S. W. Ryter is an Adjunct Scientist of the Lovelace Respiratory Research Institute (LRRI), Albuquerque, New Mexico, and he received salary support from the BWH/LRRI consortium for lung research.

\section{References}

[1] R. S. Cotran, V. Kumar, and T. Collins, Robbins Pathologic Basis of Disease, W.B. Saunders, Philadelphia, Pa, USA, 6th edition, 1999.

[2] G. Weissmann, J. E. Smolen, and H. M. Korchak, "Release of inflammatory mediators from stimulated neutrophils," The New England Journal of Medicine, vol. 303, no. 1, pp. 27-34, 1980.

[3] C. N. Serhan, "The resolution of inflammation: the devil in the flask and in the details," FASEB Journal, vol. 25, pp. 14411448, 2011.
[4] C. N. Serhan and J. Savill, "Resolution of inflammation: the beginning programs the end," Nature Immunology, vol. 6, no. 12, pp. 1191-1197, 2005.

[5] A. Luciani, V. R. Villella, S. Esposito et al., "Cystic fibrosis: a disorder with defective autophagy," Autophagy, vol. 7, no. 1, pp. 104-106, 2011.

[6] B. Levine, N. Mizushima, and H. W. Virgin, "Autophagy immunity and inflammation," Nature, vol. 469, no. 7330, pp. 323-335, 2011.

[7] A. Kelekar, "Autophagy," Annals of the New York Academy of Sciences, vol. 1066, no. 12, pp. 259-271, 2006.

[8] C. He and D. J. Klionsky, "Regulation mechanisms and signaling pathways of autophagy," Annual Review of Genetics, vol. 43, pp. 67-93, 2009.

[9] B. Levine and D. J. Klionsky, "Development by selfdigestion: molecular mechanisms and biological functions of autophagy," Developmental Cell, vol. 6, no. 4, pp. 463-477, 2004.

[10] N. Mizushima, B. Levine, A. M. Cuervo, and D. J. Klionsky, "Autophagy fights disease through cellular self-digestion," Nature, vol. 451, no. 7182, pp. 1069-1075, 2008.

[11] D. J. Klionsky and S. D. Emr, "Autophagy as a regulated pathway of cellular degradation," Science, vol. 290, no. 5497, pp. 1717-1721, 2000.

[12] T. Yorimitsu and D. J. Klionsky, "Autophagy: molecular machinery for self-eating," Cell Death and Differentiation, vol. 12, no. 2, pp. 1542-1552, 2005.

[13] H. W. Virgin and B. Levine, "Autophagy genes in immunity," Nature Immunology, vol. 10, no. 5, pp. 461-470, 2009.

[14] B. Ravikumar, S. Sarkar, J. E. Davies et al., "Regulation of mammalian autophagy in physiology and pathophysiology," Physiological Reviews, vol. 90, no. 4, pp. 1383-1435, 2010.

[15] E. L. Eskelinen and P. Saftig, "Autophagy: a lysosomal degradation pathway with a central role in health and disease," Biochimica et Biophysica Acta, vol. 1793, no. 4, pp. 664-673, 2009.

[16] Z. Yang and D. J. Klionsky, "Mammalian autophagy: core molecular machinery and signaling regulation," Current Opinion in Cell Biology, vol. 22, no. 2, pp. 124-131, 2010.

[17] X. H. Liang, S. Jackson, M. Seaman et al., "Induction of autophagy and inhibition of tumorigenesis by beclin 1," Nature, vol. 402, no. 6762, pp. 672-676, 1999.

[18] T. Johansen and T. Lamark, "Selective autophagy mediated by autophagic adapter proteins," Autophagy, vol. 7, no. 3, pp. 279-296, 2011.

[19] G. Kroemer and B. Levine, "Autophagic cell death: the story of a misnomer," Nature Reviews Molecular Cell Biology, vol. 9, no. 12, pp. 1004-1010, 2008.

[20] L. Galluzzi, J. M. Vicencio, O. Kepp, E. Tasdemir, M. C. Maiuri, and G. Kroemer, "To die or not to die: that is the autophagic question," Current Molecular Medicine, vol. 8, no. 2, pp. 78-91, 2008.

[21] M. C. Maiuri, E. Zalckvar, A. Kimchi, and G. Kroemer, "Self-eating and self-killing: crosstalk between autophagy and apoptosis," Nature Reviews Molecular Cell Biology, vol. 8, no. 9, pp. 741-752, 2007.

[22] C. H. Jung, S. H. Ro, J. Cao, N. M. Otto, and D. H. Kim DoHyung, "mTOR regulation of autophagy," FEBS Letters, vol. 584, no. 7, pp. 1287-1295, 2010.

[23] I. G. Ganley, D. H. Lam, J. Wang, X. Ding, S. Chen, and X. Jiang, "ULK1·ATG13·FIP200 complex mediates mTOR signaling and is essential for autophagy," Journal of Biological Chemistry, vol. 284, no. 18, pp. 12297-12305, 2009. 
[24] C. H. Jung, C. B. Jun, S. H. Ro et al., "ULK-Atg13-FIP200 complexes mediate mTOR signaling to the autophagy machinery," Molecular Biology of the Cell, vol. 20, no. 7, pp. 1992-2003, 2009.

[25] C. A. Mercer, A. Kaliappan, and P. B. Dennis, "A novel, human Atg13 binding protein, Atg101, interacts with ULK1 and is essential for macroautophagy," Autophagy, vol. 5, no. 5, pp. 649-662, 2009.

[26] E. Y. W. Chan, S. Kir, and S. A. Tooze, "siRNA screening of the kinome identifies ULK1 as a multidomain modulator of autophagy," Journal of Biological Chemistry, vol. 282, no. 35, pp. 25464-25474, 2007.

[27] N. Hosokawa, T. Hara, T. Kaizuka et al., "Nutrient-dependent mTORCl association with the ULK1-Atg13-FIP200 complex required for autophagy," Molecular Biology of the Cell, vol. 20, no. 7, pp. 1981-1991, 2009.

[28] C. He and B. Levine, "The Beclin 1 interactome," Current Opinion in Cell Biology, vol. 22, no. 2, pp. 140-149, 2010.

[29] S. J. Lee, A. Smith, L. Guo et al., "Autophagic protein LC3B confers resistance against hypoxia-induced pulmonary hypertension," American Journal of Respiratory and Critical Care Medicine, vol. 183, no. 5, pp. 649-658, 2011.

[30] Y. Ohsumi, "Molecular dissection of autophagy: two ubiquitin-like systems," Nature Reviews Molecular Cell Biology, vol. 2, no. 3, pp. 211-216, 2001.

[31] I. Tanida, T. Ueno, and E. Kominami, "LC3 conjugation system in mammalian autophagy," International Journal of Biochemistry and Cell Biology, vol. 36, no. 12, pp. 2503-2518, 2004.

[32] Y. Kabeya, N. Mizushima, T. Ueno et al., "LC3, a mammalian homologue of yeast Apg8p, is localized in autophagosome membranes after processing," EMBO Journal, vol. 19, no. 21, pp. 5720-5728, 2000.

[33] Y. Kabeya, N. Mizushima, A. Yamamoto, S. OshitaniOkamoto, Y. Ohsumi, and T. Yoshimori, "LC3, GABARAP and GATE16 localize to autophagosomal membrane depending on form-II formation," Journal of Cell Science, vol. 117, no. 13, pp. 2805-2812, 2004.

[34] M. G. Gutierrez, D. B. Munafó, W. Berón, and M. I. Colombo, "Rab7 is required for the normal progression of the autophagic pathway in mammalian cells," Journal of Cell Science, vol. 117, no. 6, part 13, pp. 2687-2697, 2004.

[35] S. Jäger, C. Bucci, I. Tanida et al., "Role for Rab7 in maturation of late autophagic vacuoles," Journal of Cell Science, vol. 117, no. 9, part 20, pp. 4837-4848, 2004.

[36] S. Barth, D. Glick, and K. F. Macleod, "Autophagy: assays and artifacts," Journal of Pathology, vol. 221, no. 2, pp. 117-124, 2010.

[37] P. Boya, R. A. González-Polo, N. Casares et al., "Inhibition of macroautophagy triggers apoptosis," Molecular and Cellular Biology, vol. 25, no. 3, pp. 1025-1040, 2005.

[38] S. Bertin, M. Samson, C. Pons et al., "Comparative proteomics study reveals that bacterial CpG motifs induce tumor cell autophagy in vitro and in vivo," Molecular and Cellular Proteomics, vol. 7, no. 12, pp. 2311-2322, 2008.

[39] M. A. Delgado, R. A. Elmaoued, A. S. Davis, G. Kyei, and V. Deretic, "Toll-like receptors control autophagy," EMBO Journal, vol. 27, no. 7, pp. 1110-1121, 2008.

[40] Y. Xu, C. Jagannath, X. D. Liu, A. Sharafkhaneh, K. E. Kolodziejska, and N. T. Eissa, "Toll-like receptor 4 is a sensor for autophagy associated with innate immunity," Immunity, vol. 27, no. 1, pp. 135-144, 2007.
[41] T. Saitoh, N. Fujita, M. H. Jang et al., "Loss of the autophagy protein Atg16L1 enhances endotoxin-induced IL-1 $\beta$ production," Nature, vol. 456, no. 7219, pp. 264-268, 2008.

[42] T. Saitoh, N. Fujita, T. Hayashi et al., "Atg9a controls dsDNAdriven dynamic translocation of STING and the innate immune response," Proceedings of the National Academy of Sciences of the United States of America, vol. 106, no. 49, pp. 20842-20846, 2009.

[43] M. C. Tal, M. Sasai, H. K. Lee, B. Yordy, G. S. Shadel, and A. Iwasaki, "Absence of autophagy results in reactive oxygen species-dependent amplification of RLR signaling," Proceedings of the National Academy of Sciences of the United States of America, vol. 106, no. 8, pp. 2774-2775, 2009.

[44] H. K. Lee, J. M. Lund, B. Ramanathan, N. Mizushima, and A. Iwasaki, "Autophagy-dependent viral recognition by plasmacytoid dendritic cells," Science, vol. 315, no. 5817, pp. 1398-1401, 2007.

[45] Z. Zhao, B. Fux, M. Goodwin et al., "Autophagosomeindependent essential function for the autophagy protein Atg5 in cellular immunity to intracellular pathogens," Cell Host and Microbe, vol. 4, no. 5, pp. 458-469, 2008.

[46] K. Nakahira, J. A. Haspel, V. A. K. Rathinam et al., "Autophagy proteins regulate innate immune responses by inhibiting the release of mitochondrial DNA mediated by the NALP3 inflammasome," Nature Immunology, vol. 12, pp. 222-230, 2011.

[47] R. Zhou, A. S. Yazdi, P. Menu, and J. Tschopp, "A role for mitochondria in NLRP3 inflammasome activation," Nature, vol. 469, no. 7329, pp. 221-225, 2011.

[48] K. Schroder and J. Tschopp, “The inflammasomes," Cell, vol. 140, no. 6, pp. 821-832, 2010.

[49] V. L. Crotzer and J. S. Blum, "Autophagy and adaptive immunity," Immunology, vol. 131, no. 1, pp. 9-17, 2010.

[50] D. Schmid, M. Pypaert, and C. Münz, "Antigen-loading compartments for major histocompatibility complex class II molecules continuously receive input from autophagosomes," Immunity, vol. 26, no. 1, pp. 79-92, 2007.

[51] Y. Li, L. X. Wang, G. Yang, F. Hao, W. J. Urba, and H. M. Hu, "Efficient cross-presentation depends on autophagy in tumor cells," Cancer Research, vol. 68, no. 17, pp. 6889-6895, 2008.

[52] L. English, M. Chemali, J. Duron et al., "Autophagy enhances the presentation of endogenous viral antigens on MHC class I molecules during HSV-1 infection," Nature Immunology, vol. 10, no. 5, pp. 480-487, 2009.

[53] C. Paludan, D. Schmid, M. Landthaler et al., "Endogenous MHC class II processing of a viral nuclear antigen after autophagy," Science, vol. 307, no. 5709, pp. 593-596, 2005.

[54] H. K. Lee, L. M. Mattei, B. E. Steinberg et al., "In vivo requirement for Atg5 in antigen presentation by dendritic cells," Immunity, vol. 32, no. 2, pp. 227-239, 2010.

[55] J. Nedjic, M. Aichinger, J. Emmerich, N. Mizushima, and L. Klein, "Autophagy in thymic epithelium shapes the T-cell repertoire and is essential for tolerance," Nature, vol. 455, no. 7211, pp. 396-400, 2008.

[56] D. K. Podolsky, "Inflammatory bowel disease," The New England Journal of Medicine, vol. 347, no. 6, pp. 417-429, 2002.

[57] J. Hampe, A. Franke, P. Rosenstiel et al., "A genome-wide association scan of nonsynonymous SNPs identifies a susceptibility variant for Crohn disease in ATG16L1," Nature Genetics, vol. 39, no. 2, pp. 207-211, 2007.

[58] J. D. Rioux, R. J. Xavier, K. D. Taylor et al., "Genome-wide association study identifies new susceptibility loci for Crohn 
disease and implicates autophagy in disease pathogenesis," Nature Genetics, vol. 39, no. 5, pp. 596-604, 2007.

[59] D. C. O. Massey and M. Parkes, "Genome-wide association scanning highlights two autophagy genes, ATG16L1 and IRGM, as being significantly associated with Crohn's disease," Autophagy, vol. 3, no. 6, pp. 649-651, 2007.

[60] K. Cadwell, "Crohn's disease susceptibility gene interactions, a NOD to the newcomer ATG16L1," Gastroenterology, vol. 139, no. 5, pp. 1448-1450, 2010.

[61] J. C. Barrett, S. Hansoul, D. L. Nicolae et al., "Genome-wide association defines more than 30 distinct susceptibility loci for Crohn's disease," Nature Genetics, vol. 40, no. 8, pp. 955962, 2008.

[62] M. Parkes, J. C. Barrett, N. J. Prescott et al., "Sequence variants in the autophagy gene IRGM and multiple other replicating loci contribute to Crohn's disease susceptibility," Nature Genetics, vol. 39, no. 7, pp. 830-832, 2007.

[63] C. G. Feng, C. M. Collazo-Custodio, M. Eckhaus et al., "Mice deficient in LRG-47 display increased susceptibility to mycobacterial infection associated with the induction of lymphopenia," Journal of Immunology, vol. 172, no. 2, pp. 1163-1168, 2004.

[64] S. B. Singh, A. S. Davis, G. A. Taylor, and V. Deretic, "Human IRGM induces autophagy to eliminate intracellular mycobacteria," Science, vol. 313, no. 5792, pp. 1438-1441, 2006.

[65] S. B. Singh, W. Ornatowski, I. Vergne et al., "Human IRGM regulates autophagy and cell-autonomous immunity functions through mitochondria," Nature Cell Biology, vol. 12, no. 12, pp. 1154-1165, 2010.

[66] J. P. Hugot, M. Chamaillard, H. Zouali et al., "Association of NOD2 leucine-rich repeat variants with susceptibility to Crohn's disease," Nature, vol. 411, no. 6837, pp. 599-603, 2001.

[67] J. Hampe, A. Cuthbert, P. J. P. Croucher et al., "Association between insertion mutation in NOD2 gene and Crohn's disease in German and British populations," Lancet, vol. 357, no. 9272, pp. 1925-1928, 2001.

[68] S. Lesage, H. Zouali, J. P. Cézard et al., "CARD15/NOD2 mutational analysis and genotype-phenotype correlation in 612 patients with inflammatory bowel disease," American Journal of Human Genetics, vol. 70, no. 4, pp. 845-857, 2002.

[69] T. Ahmad, A. Armuzzi, M. Bunce et al., "The molecular classification of the clinical manifestations of Crohn's disease," Gastroenterology, vol. 122, no. 4, pp. 854-866, 2002.

[70] A. P. Cuthbert, S. A. Fisher, M. M. Mirza et al., "The contribution of NOD2 gene mutations to the risk and site of disease in inflammatory bowel disease," Gastroenterology, vol. 122, no. 4, pp. 867-874, 2002.

[71] S. Vermeire, G. Wild, K. Kocher et al., "CARD15 genetic variation in a quebec population: prevalence, genotypephenotype relationship, and haplotype structure," American Journal of Human Genetics, vol. 71, no. 1, pp. 74-83, 2002.

[72] J. Hampe, J. Grebe, S. Nikolaus et al., "Association of NOD2 (CARD 15) genotype with clinical course of Crohn's disease: a cohort study," Lancet, vol. 359, no. 9318, pp. 1661-1665, 2002.

[73] M. T. Abreu, K. D. Taylor, Y. C. Lin et al., "Mutations in NOD2 are associated with fibrostenosing disease in patients with Crohn's disease," Gastroenterology, vol. 123, no. 3, pp. 679-688, 2002.

[74] W. Strober and T. Watanabe, "NOD2, an intracellular innate immune sensor involved in host defense and Crohn's disease," Mucosal Immunology, vol. 4, pp. 371-382, 2011.
[75] C. R. Homer, A. L. Richmond, N. A. Rebert, J. Achkar, and C. McDonald, "ATG16L1 and NOD2 interact in an autophagydependent antibacterial pathway implicated in crohn's disease pathogenesis," Gastroenterology, vol. 139, no. 5, pp. 1630-1641, 2010.

[76] L. H. Travassos, L. A. M. Carneiro, M. Ramjeet et al., "Nod1 and Nod2 direct autophagy by recruiting ATG16L1 to the plasma membrane at the site of bacterial entry," Nature Immunology, vol. 11, no. 1, pp. 55-62, 2010.

[77] R. Cooney, J. Baker, O. Brain et al., "NOD2 stimulation induces autophagy in dendritic cells influencing bacterial handling and antigen presentation," Nature Medicine, vol. 16, no. 1, pp. 90-97, 2010.

[78] T. S. Plantinga, T. O. Crisan, M. Oosting et al., "Crohn's disease-associated ATG16L1 polymorphism modulates proinflammatory cytokine responses selectively upon activation of NOD2," Gut, vol. 60, no. 9, pp. 1229-1235, 2011.

[79] I. Nakagawa, A. Amano, N. Mizushima et al., "Autophagy defends cells against invading group A Streptococcus," Science, vol. 306, no. 5698, pp. 1037-1040, 2004.

[80] M. G. Gutierrez, S. S. Master, S. B. Singh, G. A. Taylor, M. I. Colombo, and V. Deretic, "Autophagy is a defense mechanism inhibiting BCG and Mycobacterium tuberculosis survival in infected macrophages," Cell, vol. 119, no. 6, pp. 753-766, 2004.

[81] R. S. Flannagan, G. Cosío, and S. Grinstein, "Antimicrobial mechanisms of phagocytes and bacterial evasion strategies," Nature Reviews Microbiology, vol. 7, no. 5, pp. 355-366, 2009.

[82] F. R. Zhang, W. Huang, S. M. Chen et al., "Genomewide association study of leprosy," The New England Journal of Medicine, vol. 361, no. 27, pp. 2609-2618, 2009.

[83] H. Gan, J. Lee, F. Ren, M. Chen, H. Kornfeld, and H. G. Remold, "Mycobacterium tuberculosis blocks crosslinking of annexin-1 and apoptotic envelope formation on infected macrophages to maintain virulence," Nature Immunology, vol. 9, no. 10, pp. 1189-1197, 2008.

[84] V. Deretic, M. Delgado, I. Vergne et al., "Autophagy in immunity against mycobacterium tuberculosis: a model system to dissect immunological roles of autophagy," Current Topics in Microbiology and Immunology, vol. 335, no. 1, pp. 169-188, 2009.

[85] C. Jagannath, D. R. Lindsey, S. Dhandayuthapani, Y. Xu, R. L. Hunter, and N. T. Eissa, "Autophagy enhances the efficacy of BCG vaccine by increasing peptide presentation in mouse dendritic cells," Nature Medicine, vol. 15, no. 3, pp. 267-276, 2009.

[86] R. A. Floto, S. Sarkar, E. O. Perlstein, B. Kampmann, S. L. Schreiber, and D. C. Rubinsztein, "Small molecule enhancers of rapamycin-induced TOR inhibition promote autophagy, reduce toxicity in Huntington's disease models and enhance killing of mycobacteria by macrophages (Autophagy)," Autophagy, vol. 3, no. 6, pp. 620-622, 2007.

[87] M. Ponpuak, A. S. Davis, E. A. Roberts et al., "Delivery of cytosolic components by autophagic adaptor protein p62 endows autophagosomes with unique antimicrobial properties," Immunity, vol. 32, no. 3, pp. 329-341, 2010.

[88] J. D. MacMicking, G. A. Taylor, and J. D. McKinney, "Immune control of tuberculosis by IFN- $\gamma$-inducible LRG-47," Science, vol. 302, no. 5645, pp. 654-659, 2003.

[89] N. Che, S. Li, T. Gao et al., "Identification of a novel IRGM promoter single nucleotide polymorphism associated with tuberculosis," Clinica Chimica Acta, vol. 411, no. 21-22, pp. 1645-1649, 2010. 
[90] K. Y. King, J. D. Lew, N. P. Ha et al., "Polymorphic allele of human irgm1 is associated with susceptibility to tuberculosis in african americans," PloS One, vol. 6, no. 1, Article ID e16317, 2011.

[91] S. Alonso, K. Pethe, D. G. Russell, and G. E. Purdy, "Lysosomal killing of Mycobacterium mediated by ubiquitinderived peptides is enhanced by autophagy," Proceedings of the National Academy of Sciences of the United States of America, vol. 104, no. 14, pp. 6031-6036, 2007.

[92] L. Jordao and O. V. Vieira, "Tuberculosis: new aspects of an old disease," International Journal of Cell Biology, vol. 2011, Article ID 403623, pp. 1-13, 2011.

[93] S. M. Tung, C. Ünal, A. Ley et al., "Loss of Dictyostelium ATG9 results in a pleiotropic phenotype affecting growth, development, phagocytosis and clearance and replication of Legionella pneumophila," Cellular Microbiology, vol. 12, no. 6, pp. 765-780, 2010.

[94] J. F. Dubuisson and M. S. Swanson, "Mouse infection by Legionella, a model to analyze autophagy," Autophagy, vol. 2, no. 3, pp. 179-182, 2006.

[95] M. S. Dorer and R. R. Isberg, "Non-vertebrate hosts in the analysis of host-pathogen interactions," Microbes and Infection, vol. 8, no. 6, pp. 1637-1646, 2006.

[96] S. Bozzaro, C. Bucci, and M. Steinert, "Chapter 6 phagocytosis and host-pathogen interactions in dictyostelium with a look at macrophages," International Review of Cell and Molecular Biology, vol. 271, pp. 253-300, 2008.

[97] T. Jin, X. Xu, J. Fang et al., "How human leukocytes track down and destroy pathogens: lessons learned from the model organism Dictyostelium discoideum," Immunologic Research, vol. 43, no. 1-3, pp. 118-127, 2009.

[98] J. Calvo-Garrido, S. Carilla-Latorre, Y. Kubohara et al., "Autophagy in dictyostelium: genes and pathways, cell death and infection," Autophagy, vol. 6, no. 6, pp. 686-701, 2010.

[99] M. Steinert and K. Heuner, "Dictyostelium as host model for pathogenesis," Cellular Microbiology, vol. 7, no. 3, pp. 307314, 2005.

[100] G. P. Otto, M. Y. Wu, N. Kazgan, O. R. Anderson, and R. H. Kessin, "Macroautophagy is required for multicellular development of the social amoeba Dictyostelium discoideum," Journal of Biological Chemistry, vol. 278, no. 20, pp. 1763617645, 2003.

[101] G. P. Otto, M. Y. Wu, N. Kazgan, O. R. Anderson, and R. H. Kessin, "Dictyostelium macroautophagy mutants vary in the severity of their developmental defects," Journal of Biological Chemistry, vol. 279, no. 15, pp. 15621-15629, 2004.

[102] S. M. Tung, C. Ünal, A. Ley et al., "Loss of Dictyostelium ATG9 results in a pleiotropic phenotype affecting growth, development, phagocytosis and clearance and replication of Legionella pneumophila," Cellular Microbiology, vol. 12, no. 6, pp. 765-780, 2010.

[103] G. P. Otto, M. Y. Wu, M. Clarke et al., "Macroautophagy is dispensable for intracellular replication of Legionella pneumophila in Dictyostelium discoideum," Molecular Microbiology, vol. 51, no. 1, pp. 63-72, 2004.

[104] R. A. Balk, "Severe sepsis and septic shock: definitions, epidemiology, and clinical manifestations," Critical Care Clinics, vol. 16, no. 2, pp. 179-192, 2000.

[105] E. Watanabe, J. T. Muenzer, W. G. Hawkins et al., "Sepsis induces extensive autophagic vacuolization in hepatocytes: a clinical and laboratory-based study," Laboratory Investigation, vol. 89, no. 5, pp. 549-561, 2009.
[106] S. Y. Chan and J. Loscalzo, "Pathogenic mechanisms of pulmonary arterial hypertension," Journal of Molecular and Cellular Cardiology, vol. 44, no. 1, pp. 14-30, 2008.

[107] R. M. Tuder, S. H. Abman, T. Braun et al., "Development and pathology of pulmonary hypertension," Journal of the American College of Cardiology, vol. 54, no. 1, pp. S3-S9, 2009.

[108] F. Ratjen and G. Döring, "Cystic fibrosis," Lancet, vol. 361, no. 9358, pp. 681-689, 2003.

[109] A. Luciani, V. R. Villella, S. Esposito et al., "Defective CFTR induces aggresome formation and lung inflammation in cystic fibrosis through ROS-mediated autophagy inhibition," Nature Cell Biology, vol. 12, no. 9, pp. 863-875, 2010.

[110] S. W. Ryter and A. M. Choi, "Autophagy in the lung," Proceedings of the American Thoracic Society, vol. 7, no. 1, pp. 13-21, 2010.

[111] S. W. Ryter, S. J. Lee, A. Smith, and A. M. Choi, "Autophagy in vascular disease," Proceedings of the American Thoracic Society, vol. 7, no. 1, pp. 40-47, 2010. 

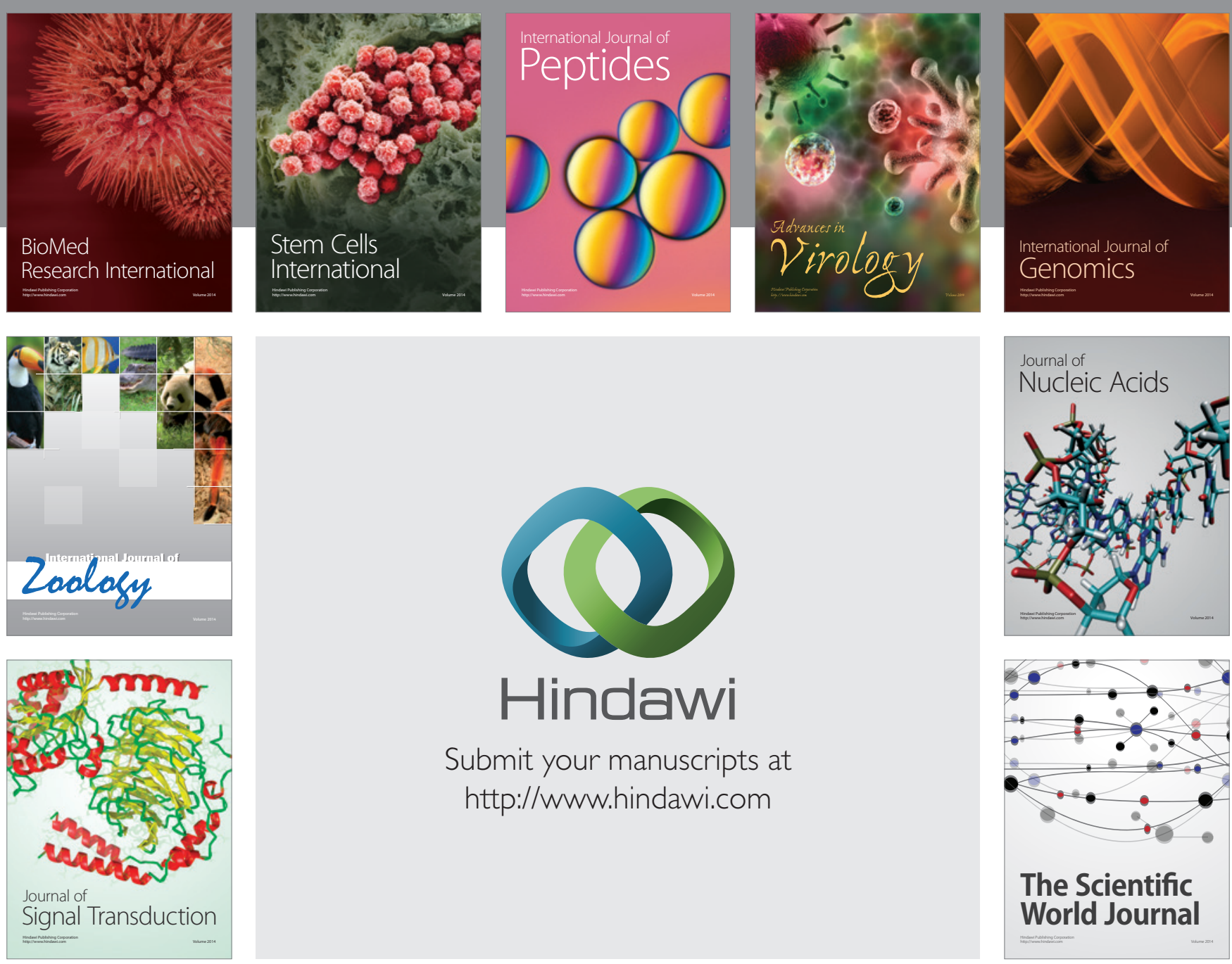

Submit your manuscripts at

http://www.hindawi.com
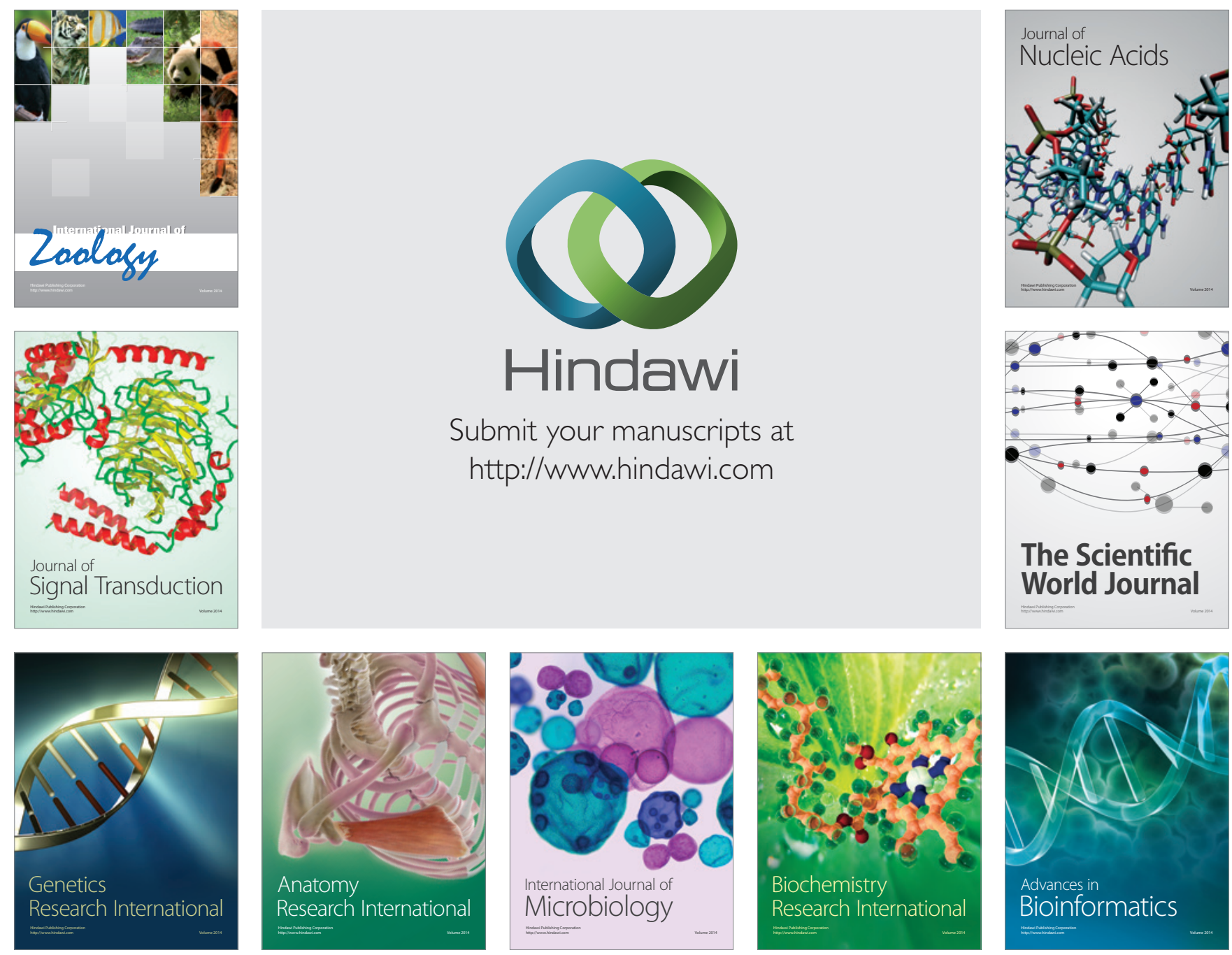

The Scientific World Journal
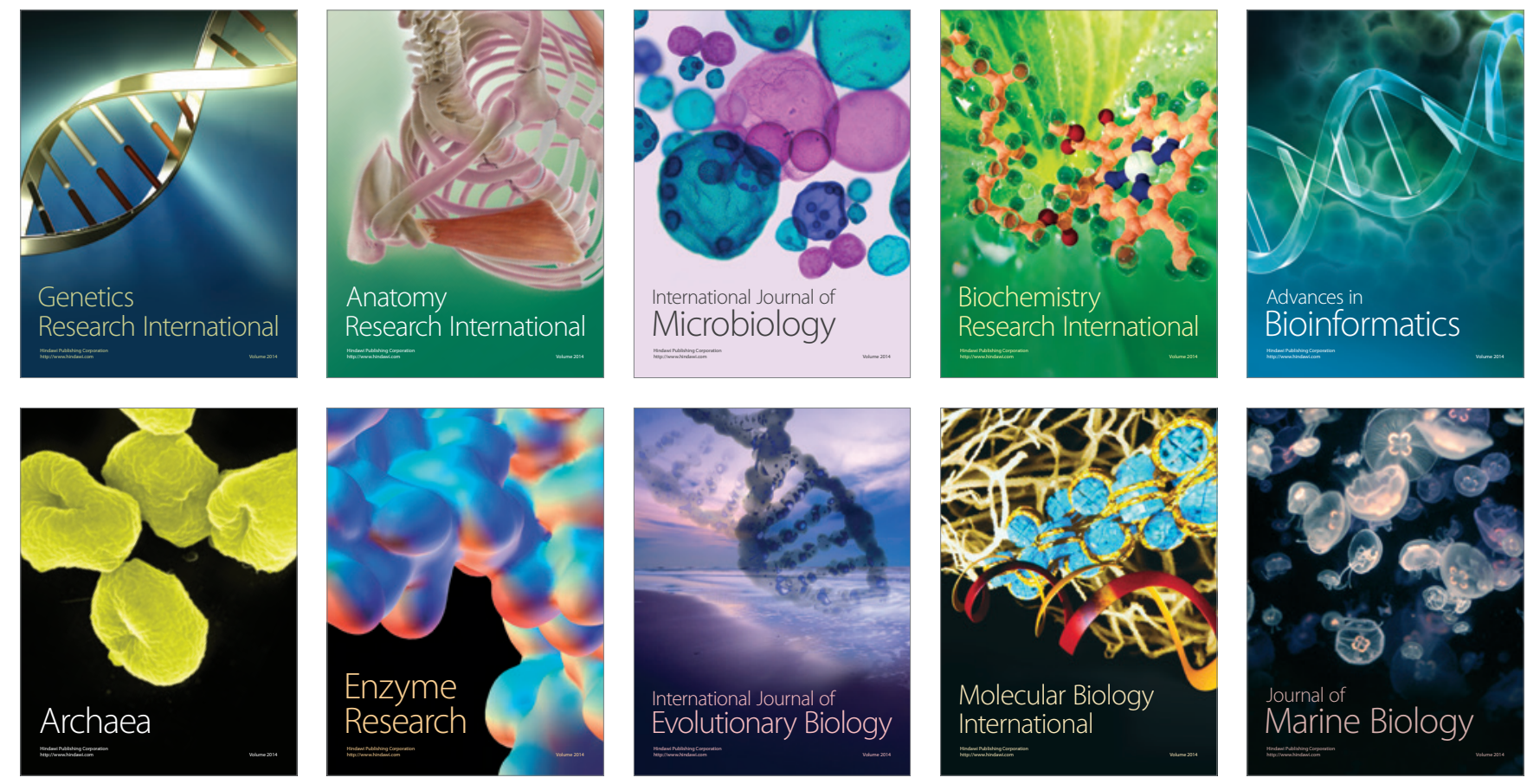